\title{
Title: Autonomous Microstructure EM-APEX Floats
}

Authors: Ren-Chieh Lien ${ }^{1,2}$, Thomas B. Sanford ${ }^{1,2}$, James A. Carlson ${ }^{1}$, and John H. Dunlap ${ }^{1}$

${ }^{1}$ Applied Physics Laboratory, University of Washington, Seattle, WA

${ }^{2}$ School of Oceanography, University of Washington, Seattle, WA

Corresponding author:

Ren-Chieh Lien

Applied Physics Laboratory, University of Washington

1013 NE $40^{\text {th }}$ Street

Seattle, WA 98105

rcl@uw.edu 


\begin{abstract}
:
Fast responding FP-07 thermistors have been incorporated on autonomous profiling EM-APEX floats to measure microscale ocean temperature fluctuations produced by turbulence. In this implementation, the FP-07 thermistor generates an electrical signal corresponding to ocean temperature fluctuations, which is conditioned by an analog circuit board, and digitized and recorded on a custom data acquisition and storage board. The raw and processed temperature observations are stored on a microSD card. Results from eight microstructure EM-APEX floats deployed in the Sargasso Sea are presented here. The slow profiling speed of EM-APEX floats enables them to resolve the higher wavenumber regime of the microscale temperature gradient spectrum, beyond the roll-off wavenumber. The temperature variance dissipation rate $\chi$ is computed directly by integrating the observed temperature gradient spectrum over the turbulence wavenumber region without the need to fit the observed temperature gradient spectrum to the empirical spectral form. The accuracy of $\chi$ estimated from microstructure EM-APEX floats is confirmed by the agreement, within a factor of 2 , between the temperature diffusivity $K_{T}$ computed from our estimates of $\chi$ and estimates of diapcynal diffusivity computed from simultaneous tracer measurements. The observed temperature gradient spectra averaged over many realizations resemble the Batchelor spectral form, though individual spectra often do not fit the empirical prediction. Estimates of $\chi$ from different floats have similar temporal fluctuations and vertical profiles, further supporting measurement quality. Estimates of $\chi$ exhibit a lognormal distribution, as expected for statistically homogeneous isotropic turbulence. Turbulence measurements derived from FP-07 sensors on autonomous profiling floats are of comparable quality to those on conventional free-fall microstructure profilers.
\end{abstract}




\section{Introduction}

Quantifying oceanic turbulence, diffusion, and dissipation gives insight to important oceanic processes and improves parameterization schemes in numerical models. The intermittency of turbulence and the difficulty obtaining adequate realizations of turbulence observations limit the reliability of turbulence estimations. One sensor, the FP-07 thermistor, is capable of rapid temperature sampling at microscales, and has been used extensively on free-fall vertical profilers and moorings (Gregg and Meagher, 1980; Moum and Nash, 2009). FP-07 thermistors have also been implemented on autonomous floats where they obtained quality turbulence data (Sherman and Davis, 1995). The temperature variance dissipation rate $\chi$ can be computed from FP-07 microscale temperature measurements. The development of autonomous vertical profilers and the ability to deploy several in an array allows collection of many profiles at multiple sites simultaneously. Because of the dynamical link between turbulence and small-scale vertical shear and strain, autonomous profiling floats with FP-07 thermistors, as well as other sensors to observe conventional water properties, are ideal to better measure and understand small-scale and microscale processes and improve physics-based turbulence parameterization schemes. Here, we describe our approach to implement turbulence sensing on the EM-APEX float and present results from deployments of multiple floats.

\section{Material and methods}

\subsection{EM-APEX floats}

The EM-APEX float combines the standard Teledyne Webb Research Corp. APEX profiling float with an APL-UW developed subsystem that measures the motionally induced electric fields generated by the ocean currents moving through the vertical component of the earth's magnetic 
Autonomous Microstructure_EM-APEX_Float 10/17/17 at 10:31 PM

field (Fig. 1) (Sanford et al., 2005). The APEX float changes its buoyancy to enable it to profile the ocean with a maximum depth range of $2000 \mathrm{~m}$. Temperature and salinity measurements are taken by a Sea-Bird Electronics SBE41CP CTD every 10-25 s. When on the sea surface, the float's position is determined by GPS, and the position and accumulated profile data (except for the raw FP-07 data) are transmitted over the Iridium global satellite communications system.

The velocity sensor operates on the same principles of motional induction applied on the Absolute Velocity Profiler (Sanford et al., 1985) and the Expendable Current Profiler (Sanford et al., 1982). The electric field sensing electrodes are located on the top end of the floats (Fig. 1). Other necessary measurements are magnetic compass heading and instrument tilt. The float descends and ascends at $\sim 0.15 \mathrm{~m} \mathrm{~s}^{-1}$ and rotates at a period of $\sim 12 \mathrm{~s}$. The motional-induced electric field is determined by a sinusoidal fit to the measured voltages using the basis functions from the horizontal components of the magnetometer. The fit is made over a 50-s-long segment of data, and the averaging window is moved $25 \mathrm{~s}$ between successive fits. These sinusoidal fits and the rms (root mean square) residuals are transferred to the APEX float controller for storage and later transmission over Iridium. The fits provide an estimate of the horizontal current, and the rms residuals provide an estimate of the velocity noise level. The horizontal current uncertainty is about $0.015 \mathrm{~m} \mathrm{~s}^{-1}$. In the upper ocean, the rms residuals can also be used to estimate properties of surface gravity waves, e.g., amplitude, period, and wavelength.

\subsection{Turbulence sensor implementation}

A fast-response temperature sensor, the FP-07 thermistor, and electronic signal conditioning board are provided by Rockland Scientific, Inc. (RSI), and combined with a custom digital data acquisition board. The complete unit is installed in EM-APEX floats (Fig. 1). In addition to the 
Autonomous Microstructure_EM-APEX_Float 10/17/17 at 10:31 PM

thermistors installed in metal shafts or stings, the implementation requires special end caps for the EM-APEX float.

The arrangement of the major components within the EM-APEX float is outlined in a block diagram (Fig. 2). Temperature fluctuations cause the FP-07 thermistor to change its resistance. Electric current passing through the FP-07 thermistor produces voltage fluctuations caused by these resistance fluctuations. The voltages are amplified and conditioned on the RSI $\mu$ ASTP-LP board and passed to the APL-UW board. The APL-UW board (MLADC) digitizes the amplified voltage signals to 24-bit resolution. The board also contains a low-power microprocessor that buffers and stores the digital data onto a microSD card. In the latest version of the microstructure package, temperature spectra are computed and band-averaged spectra are transmitted over Iridium. The turbulence circuitry is powered and controlled by the APEX controller APF-9i board.

2.3 Estimates of temperature variance dissipation rate $\chi$ and turbulent temperature diffusivity $K_{T}$

The high-frequency FP-07 sensor provides measurements of turbulent temperature fluctuations that can be used to compute the temperature variance dissipation rate $\chi$.The temperature variance dissipation rate for isotropic turbulence is defined as

$$
\chi=2 D_{T}\left\langle\left(\frac{\partial T}{\partial x}\right)^{2}+\left(\frac{\partial T}{\partial y}\right)^{2}+\left(\frac{\partial T}{\partial z}\right)^{2}\right\rangle=6 D_{T}\left\langle\left(\frac{\partial T}{\partial z}\right)^{2}\right\rangle=6 D_{T} \int_{k_{o}}^{\infty} \phi_{\partial_{z} T}\left(k_{z}\right) d k_{z}
$$

where $D_{T}$ is the molecular temperature diffusivity $\left(\sim 1.4 \times 10^{-7} \mathrm{~m}^{2} \mathrm{~s}^{-1}\right), \phi_{\partial_{z} T}\left(k_{z}\right)$ the vertical wavenumber $k_{z}$ spectrum of the vertical gradient of temperature $\frac{\partial T}{\partial z},\langle\cdot\rangle$ the average over the turbulence scale, and $k_{o}$ the lowest wavenumber of significant turbulence. 
Autonomous Microstructure_EM-APEX_Float 10/17/17 at 10:31 PM

Three transfer functions are applied to FP-07 sensor measurements to derive the temperature gradient spectrum; they are the analog-to-digital conversion $H_{\mathrm{ADC}}$, amplification of the highfrequency component $H_{\text {Preamp }}$, and the response of the sensor to temperature fluctuations $H_{\mathrm{FP} 07}$, where $H$ represents the transfer function. The analytical forms of the transfer functions $H_{\mathrm{ADC}}$ and $H_{\text {Preamp }}$ are provided by manufacturers Cirrus Logic and Rockland Scientific, respectively.

The transfer function $H_{\mathrm{FP} 07}$ is not known exactly and different findings have been reported (Sherman and Davis 1995; Nash et al. 1999; Sommer et al. 2013). We examine the effects on our estimates of $\chi$ of three double-pole transfer functions proposed by Vachon and Lueck (1984), Gregg and Meagher (1980), and Sommer et al. (2013). The squared double-pole transfer function $\left(H_{F P 07}^{2}\right)$ is expressed as

$$
H_{F P 07}(f)^{2}=\frac{1}{\left[1+(2 \pi f \tau)^{2}\right]^{2}}
$$

where $f$ is the frequency in $\mathrm{Hz}$ and $\tau$ is a constant characterizing the time in seconds of FP-07 sensor response to ambient temperature fluctuation. The time constant is suggested to vary with the flow speed relative to the sensor expressed as,

$$
\tau=\tau_{0}\left(\frac{U}{U_{0}}\right)^{S}
$$

where $\tau_{0}$ is a reference time constant, and $U$ the flow speed relative to the sensor in $\mathrm{m} \mathrm{s}^{-1}$ and $U_{0}$ $=1 \mathrm{~m} \mathrm{~s}^{-1}$. The reference time $\tau_{0}$ is $0.0064 \mathrm{~s}, 0.005 \mathrm{~s}$, and $0.010 \mathrm{~s}$, and the speed-dependence power law exponent $(s)$ is $-0.5,-0.32$, and 0 for the transfer functions suggested by Vachon and Lueck (1984), Gregg and Meagher (1980), and Sommer et al. (2013), respectively. At the typical EM-APEX float vertical profiling speed of $0.15 \mathrm{~m} \mathrm{~s}^{-1}$, the time constant $\tau$ is $0.0155 \mathrm{~s}, 0.0092 \mathrm{~s}$, and $0.010 \mathrm{~s}$ for the Vachon and Lueck (1984), Gregg and Meagher (1980), and Sommer et al. 
Autonomous Microstructure_EM-APEX_Float 10/17/17 at 10:31 PM

(2013) transfer functions, respectively. The latter two transfer functions are in very close agreement.

FP-07 sensors are mounted on the top end of EM-APEX floats, so measurements are taken only during ascending profiles to avoid wake effects. The FP-07 samples temperature at $120 \mathrm{~Hz}$. Estimates of $\chi$ were computed from 4096 temperature data points over $~ 34$-s duration, corresponding to $\sim 5-\mathrm{m}$ vertical bin for a typical EM-APEX float ascending speed of $0.15 \mathrm{~m} \mathrm{~s}^{-1}$.

Computing $\chi$ from one time series of 34-s duration begins with the voltage measured by the FP-07 converted to temperature, then calibrated with the standard SBE41CP CTD sensor on the float (Fig. 3a). The frequency spectrum of temperature is computed and converted to the vertical wavenumber spectrum assuming a frozen hypothesis using the average ascending float speed within the 34-s time interval (Fig. 3b). The vertical temperature gradient spectrum is computed as vertical wavenumber squared times the vertical wavenumber temperature spectrum (Fig. 3c). The vertical temperature gradient spectrum is then corrected by the above-mentioned three transfer functions (Fig. 3e). The $H_{\mathrm{ADC}}$ squared is white at low wavenumbers and rolls off beyond $\sim 5 \times 10^{2} \mathrm{~m}^{-1}$. The $H_{\text {Preamp }}$ squared is white at the lowest wavenumbers, has a +2 spectral slope beyond $\sim 10 \mathrm{~m}^{-1}$. Transfer functions proposed by Sommer et al. (1984) and Gregg and Meagher (1980) are in close agreement, but that proposed by Vachon and Lueck (1984) rolls off at a wavenumber about 30\% less (Fig. 3d).

The vertical temperature gradient spectra corrected by the three $H_{\mathrm{FP} 07}$ transfer functions are in close agreement (Fig. 3e-f). Because of the low profiling speed, the roll-off spectral shape of the temperature gradient spectrum is fully resolved, implying that most turbulent temperature gradient variance is captured. The noise spectrum shows a spectral shape of $k_{z}{ }^{2}$ at wavenumber greater than $10^{3} \mathrm{~m}^{-1}$. 
Estimates of $\chi$ are computed by integrating the temperature gradient spectrum from $k_{z}=12$ $\mathrm{m}^{-1}$ to an upper bound $k_{z}$ beyond which the observed temperature gradient spectrum is less than the noise spectrum. The lower bound wavenumber $12 \mathrm{~m}^{-1}$ was determined after examining observed spectra. Estimated $\chi$ in this example is $4.9 \times 10^{-9} \mathrm{~K}^{2} \mathrm{~s}^{-1}$ using the FP-07 transfer function of Vachon and Lueck (1984) and $3.6 \times 10^{-9} \mathrm{~K}^{2} \mathrm{~s}^{-1}$ using those of Gregg and Meagher (1980) and Sommer et al. (2013). On average, estimates of $\chi$ using the latter two transfer functions differ by less than $2 \%$, and are $35 \%$ less than those estimated using the transfer function of Vachon and Lueck (1984).

Note that because the full turbulent temperature gradient spectrum is resolved, estimates of $\chi$ are computed by directly integrating the observed temperature gradient spectrum over the turbulence range without the assumption of a model temperature gradient spectrum, as proposed by Batchelor (1959) or Kraichnan (1967). In fact, observed individual temperature gradient spectra often differ from the empirical prediction. However, observed temperature gradient spectra averaged over many realizations are in good agreement with the Batchelor (1959) temperature spectrum (section 3.2).

The turbulence diffusivity of temperature $K_{T}$ can be computed following Osborn and Cox (1972) as

$$
K_{T}=\frac{\chi}{2\left(\frac{d T}{d z}\right)^{2}}
$$

where $\left\langle\frac{d T}{d z}\right\rangle$ is the background temperature gradient. In this analysis, $\left\langle\frac{d T}{d z}\right\rangle$ is defined as the temperature gradient at 5-m vertical bin.

Assuming that the turbulent diffusivity of temperature $K_{T}$ equals the turbulent diffusivity of buoyancy (e.g., Moum and Nash, 2009; Alford and Pinkel, 2000), defined as $K_{\rho}=\Gamma \varepsilon / N^{2}$, 
where $\varepsilon$ is the turbulent kinetic energy dissipation rate, $N$ the buoyancy frequency, and $\Gamma$ the mixing efficiency assumed as the nominal value of 0.2 , we can estimate $\varepsilon=\frac{N^{2} \chi}{2 \Gamma\left(\frac{d T}{d z}\right)^{2}}$. Note that in this analysis estimates of $\varepsilon=\frac{N^{2} \chi}{2 \Gamma\left(\frac{d T}{d z}\right\rangle^{2}}$, and thereby the assumption of $K_{T}=K_{\rho}$, are used only to construct the Batchelor temperature gradient spectrum (1959) to compare with the observed spectra. Our estimates of $\chi$ and $K_{T}$ do not require the assumption of $K_{T}=K_{\rho}$ and estimates of $\varepsilon$.

\section{Results}

\subsection{Experimental observations}

Twenty EM-APEX floats were deployed in the Sargasso Sea southeast of Cape Hatteras in 2011 as part of the ONR-funded Scalable Lateral Mixing and Coherent Turbulence (short title: LatMix) Departmental Research Initiative (Fig. 4) (Shcherbina et al., 2015). All 20 EM-APEX floats measured temperature, salinity, pressure, and horizontal velocity components between the surface and typically $150-\mathrm{m}$ depth, with some deeper profiles to $250 \mathrm{~m}$. Although the floats were at different locations, with separations up to $10 \mathrm{~km}$, the units were programmed to rise nearly in synchronism. Extensive effort was made to synchronize the profiling floats. Simultaneous profiles eliminate differences caused by temporal versus spatial differences. Ten of the EM-APEX floats were equipped with dual FP-07 sensors. One of these $\chi$-EM-APEX floats failed during the first deployment and was not used again in the experiment, and another was lost in the second deployment. Therefore, eight $\chi$-EM-APEX floats provided turbulence measurements.

Floats were deployed in three settings (Fig. 4): Deployment 1 (D1) was conducted during 3 10 June 2011 (4988 profiles), Deployment 2 (D2) during 13 - 16 June 2011 (2546 profiles), and 
Autonomous Microstructure_EM-APEX_Float 10/17/17 at 10:31 PM

Deployment 3 (D3) during 17 - 20 June 2011 (2032 profiles) (Fig. 4). D1 was conducted during a weak straining period, and D2 and D3 were conducted during a moderate straining period (Shcherbina et al., 2015). Floats were intended to be deployed in three concentric circles of 0.5 $\mathrm{km}, 1 \mathrm{~km}$, and $2 \mathrm{~km}$ radii in the first two deployments, and of $1 \mathrm{~km}, 2 \mathrm{~km}$, and $4 \mathrm{~km}$ radii in the third deployment, with roughly six floats on each circle. However, because of the oceanic horizontal shear, float arrays were not deployed in a perfectly concentric configuration.

During the 7-day mission of the first deployment, 20 EM-APEX floats were advected southeast about $20 \mathrm{~km}$ (Fig. 4). There was little dispersion of the float array. During the 3-day mission of the second deployment, the float array was strongly sheared in a front, while moved northeast about $30 \mathrm{~km}$. During the 3-day mission of the third deployment, the float array was advected northeast for $100 \mathrm{~km}$.

During the weak straining period, a 1.5-km-long zonal streak of rhodamine dye was released on 4 June at a targeted potential density of $25.42 \mathrm{~kg} \mathrm{~m}^{-3}$, with a mean depth of $\sim 30 \mathrm{~m}$. During the moderate straining period, a 2-km streak of rhodamine was released on 13 June at a targeted potential density of $25.04 \mathrm{~kg} \mathrm{~m}^{-3}$, with a mean depth of $\sim 30 \mathrm{~m}$. Evolution of the vertical distribution of rhodamine is used to compute diapycnal diffusivity. The average diapycnal diffusivity is $5 \times 10^{-6} \mathrm{~m}^{2} \mathrm{~s}^{-1}$, and $3-6 \times 10^{-6} \mathrm{~m}^{2} \mathrm{~s}^{-1}$ during the weak and moderate straining period, respectively (Shcherbina et al., 2015; Sundermeyer et al., 2016).

\subsection{Turbulence properties}

The quality of $\chi$ is assessed by three independent methods: (1) comparing the observed temperature gradient spectrum with the canonical form of the turbulent temperature gradient spectrum (Batchelor, 1959), (2) comparing estimates of $\chi$ obtained from different floats, and (3) 
comparing the statistics of $\chi$ estimates with the expectation for equilibrium turbulence. The accuracy of $K_{T}$ is assessed by comparison with the diapycnal diffusivity computed from tracer measurements taken during the same period as float deployments.

In the following analysis, estimates of $\chi$ are computed using the transfer function of Gregg and Meagher (1984). The ratio of $\chi$ estimates computed from two FP-07 sensors on the same float has a mean ratio of 1.06 and $95 \%$ confidence interval of $0.50-1.88$. Estimates of $\chi$ with the ratios between those obtained from two FP-07 sensors less a factor of two are averaged and discussed in the following analysis. Similarly, estimates of turbulent temperature diffusivity $K_{T}$ from two FP-07 sensors within a factor of two are averaged. Our estimates of $K_{T}$ are compared with those obtained from tracer measurements.

Observed temperature gradient spectra and their variance preserving spectra are compared with those of Batchelor (1959). Although some observed spectra agree with the Batchelor spectrum (Fig. 3e), many individual observed spectra do not, as reported by Sherman and Davis (1995). However, observed temperature gradient spectra averaged over many realizations do agree with Batchelor spectrum predictions (Fig. 5). Observed temperature gradient spectra of similar values of $\chi_{i}$ are averaged,

$$
\overline{\Phi_{\partial_{z} T}^{o b s}}\left(k_{z} ; \chi_{i}\right)=\frac{1}{N} \sum_{j=1}^{N} \Phi_{\partial_{z} T}^{o b s}\left(k_{z} ; \chi_{\mathrm{j}}\right),
$$

where $0.5 \leq \frac{\chi_{j}}{\chi_{i}} \leq 2, \chi_{j}$ is the estimate of $\chi$ computed from the $j^{\text {th }}$ observed spectrum, and $\chi_{i}$ are chosen as $10^{-10}, 10^{-9}, 10^{-8}, 10^{-7}$, and $10^{-6} \mathrm{~K}^{2} \mathrm{~s}^{-1}$. The averaged observed spectrum is compared with the averaged Batchelor spectrum computed as

$$
\overline{\Phi_{\partial_{z} T}^{B a t}}\left(k_{z} ; \chi_{i}\right)=\frac{1}{N} \sum_{j=1}^{N} \Phi_{\partial_{z} T}^{B a t}\left(k_{z} ; \chi_{j}, \varepsilon_{j}\right),
$$


where $\varepsilon_{j}=\frac{N^{2} \chi_{j}}{2 \Gamma\left(\frac{d T}{d z}\right)^{2}}$.

Averages of observed spectra agree well with Batchelor spectra near the roll-off wavenumbers, where most temperature gradient variance resides (Fig. 5). They differ at low wavenumbers, where the temperature gradient variance is small and therefore does not contribute significantly to the estimate of $\chi$. The observed temperature gradient spectra show a blue noise spectrum at high wavenumbers (Fig. 5a). The noise is likely due to the amplified electronic noise in the float. The noise level of our $\chi$ estimates is about $10^{-9} \mathrm{~K}^{2} \mathrm{~s}^{-1}$, where the peak of the variance is closer to the noise (Fig. 5b).

Vertical profiles of $\chi$ estimated from eight floats, averaged over each of three deployments, show similar vertical structures with their magnitudes typically within a factor of three from the means from all floats (Fig. 6). Estimates of $\chi$ obtained from different floats at different depths are strongly correlated with $\chi$ averaged over all floats with a correlation coefficient $\mathrm{R}^{2}=0.94$, much higher than the $95 \%$ significance level of 0.07 (Fig. 7). The variation is less than a factor of two for $\chi$ greater than $3 \times 10^{-9} \mathrm{~K}^{2} \mathrm{~s}^{-1}$ and a factor of six for smaller $\chi$, where the noise becomes significant (Fig. 5). The temperature signal is contaminated by electronic noise in the float. In a later version of the microstructure EM-APEX system, the preamps are relocated outside the endcap to minimize the contamination by electronic noise from within the float.

The temporal variation of depth-averaged $\chi$ observed by different floats is similar most of time (Fig. 8). The greatest variability of depth-averaged $\chi$, observed during 15-16 June, is presumably associated with a real oceanic signal of turbulence variability when the float array encountered a front (Shcherbina et al., 2015). At other times, the variability of the depth averaged $\chi$ of different floats is about a factor of two. 
For statistically homogeneous isotropic turbulence, we expect $\chi$ to have a lognormal distribution (Yaglom, 1960; Gregg et al., 1993; Yamazaki, 1990). The probability density function of $\chi$ estimates computed from all floats shows a nearly lognormal distribution, with a mean $\log _{10}(\chi)$ of -8.4 and a standard deviation of -0.9 (Fig. 9). The Quantile-Quantile (Q-Q) plot shows the quantile of observed $\log _{10}(\chi)$ vs. the standardized normal distribution (Chambers et al. 1983). The agreement is good for $\log _{10}(\chi)$ between -9.5 and -6.8 , within $\sim 1.2$ standard deviation from the mean. Yamazaki and Lueck (1990) report that when turbulence is not statistically homogeneous, the observed turbulent kinetic energy dissipation rate $\varepsilon$ might not exhibit a lognormal distribution. We suspect that the deviation of lognormal distribution at low $\chi$ is due to the measurement noise, and the deviation at high $\chi$ may be due to a violation of the assumptions of statistical homogeneity.

To examine the accuracy of our estimates of turbulent temperature diffusivity $K_{T}$ computed from $\chi$, estimates of $K_{T}$ are averaged over all floats and over the weak straining and strong straining periods, separately, along isopycnal surfaces and are compared with the diapycnal diffusivity computed from tracer measurements (Fig. 10). During the weak straining period, the diapycnal diffusivity is $5 \times 10^{-6} \mathrm{~m}^{2} \mathrm{~s}^{-1}$ computed from tracer evolution on the $25.42 \mathrm{~kg} \mathrm{~m}^{-3}$ potential density surface (Shcherbina et al., 2015; Sundermeyer et al., 2016). Averaging over the weak straining period, our estimates of $K_{T}$ on that isopycnal surface is $10^{-5} \mathrm{~m}^{2} \mathrm{~s}^{-1}$, with the $95 \%$ confidence interval between $3 \times 10^{-6}$ and $2 \times 10^{-5} \mathrm{~m}^{2} \mathrm{~s}^{-1}$ (Fig. 10). During the moderate straining period, the tracer derived diffusivity on the $25.04 \mathrm{~kg} \mathrm{~m}^{-3}$ potential density surface is $3-6 \times 10^{-6}$ $\mathrm{m}^{2} \mathrm{~s}^{-1}$. Averaging over the moderate straining period, our estimates of $K_{T}$ on that isopycnal surface is $6 \times 10^{-6} \mathrm{~m}^{2} \mathrm{~s}^{-1}$, with the $95 \%$ confidence interval between $2 \times 10^{-6}$ and $10^{-5} \mathrm{~m}^{2} \mathrm{~s}^{-1}$. 
Overall, our estimates of averaged turbulent temperature diffusivity is within of factor of two of the diapycnal diffusivity derived from tracer measurements.

\section{Discussion and conclusions}

Our assessment of the quality of $\chi$ estimates is based on three independent methods. The accuracy of turbulent temperature diffusivity is confirmed by comparing with diapycnal diffusivity obtained from tracer measurements. Estimates of $\chi$ are reliable at values greater than order of $10^{-9} \mathrm{~K}^{2} \mathrm{~s}^{-1}$. Most importantly, because of the float's slow profiling speed, the roll-off regime of the turbulence gradient spectrum is captured and therefore $\chi$ is computed by directly integrating the observed temperature gradient spectrum without fitting to the canonical Batchelor spectral form. In comparison, free-fall microstructure profilers typically fall at a speed of $0.5-1$ $\mathrm{m} \mathrm{s}^{-1}, 3-7$ times faster than the float's profiling speed, to capture turbulence shear variance measured from air-foil shear probes. Unfortunately, at this high speed, the roll-off wavenumber of the temperature gradient spectrum is often not captured by the FP-07 sensor, and therefore the observed spectrum is often fitted to the canonical Batchelor or Kraichnan spectral form to correct for the missing variance of temperature gradient at high wavenumbers.

The installation of FP-07 sensors on the eight EM-APEX floats used in the LatMix swarm was able to observe the small-scale temperature fluctuations associated with ocean turbulence. The observations conform to the expectations of the lognormal distribution of homogeneous turbulence at intermediate levels of turbulence. Significant differences in turbulence temperature dissipate rates were calculated from floats across a front. This is expected in a region of spatial gradients in oceanic properties. 
On the basis of the assessment of the quality $\chi$ estimates and the good agreement between our estimates of $K_{T}$ and the diapycnal diffusivity from tracer measurements, we conclude that the performance of the $\chi$ system was adequate for this first application on the EM-APEX floats. There were some performance limitations. A concurrent installation of the RSI FP-07 and preamp/conditioning board on Seagliders by our colleagues Luc Rainville and Jason Gobat has a much lower noise level. This suggests that our design is contaminated by the amplified EM noise inside the float. The stainless steel metal stings also contributed corrosion noise that was observed on the $\mathrm{Ag}-\mathrm{AgCl}$ electrodes near the end cap. These led to noise in the float's velocity measurements. Both of these effects have been reduced in the latest design. The thermistor preamps in the latest design are outside of the end cap, so that electronic noise within the float is not amplified and its contamination is minimized. Also, the stainless steel stings have been replaced with an all plastic holder for thermistor probes and preamps.

The need to recover the floats to extract data on the microSB card prevented the use of this design in other experiments. The latest firmware has been changed to provide log averaged spectral values. The amount of data transmitted over Iridium is expected to be about the same order as that for the CTD and velocity data. 
Autonomous Microstructure_EM-APEX_Float 10/17/17 at 10:31 PM

\section{Acknowledgements}

Avery Snyder helped with the construction of $\chi$-EM-APEX floats and supported the field operations during the LatMix experiment. We are grateful for the collaboration with Jason Gobat and Luc Rainville, especially in terms of future improvements for the implementation. We appreciate that Miles Sundermeyer kindly provides values of diapycnal diffusivity computed from tracer measurements. We thank Steve Kahle for preparing Fig. 2. Financial support was provided by the Office of Naval Research Physical Oceanography Program under grants N00014-09-0194 and N00014-01-2184 
Autonomous Microstructure_EM-APEX_Float 10/17/17 at 10:31 PM

\section{References}

Alford, M. H., and R. Pinkel (2000). Patterns of turbulent and double-diffusive phenomena: Observations from a rapid profiling micro-conductivity probe. J. Phys. Oceanogr., 30, 833854.

Batchelor, G. K. (1959). Small scale variation of convected quantities like temperature in turbulent fluid. Part 1: General discussion and the case of small conductivity. J. Fluid. Mech, 5, 113-133.

Chambers, J. M., W. S. Cleveland, B. Kleiner, and P. A. Tukey (1983). Graphical methods for data analysis. Duxbury Press, 395pp.

Gregg, M.C., and T. B. Meagher (1980). The dynamic response of glass rod thermistors. $J$. Geophys. Res., 85, 2779-2786.

Gregg, M. C., H. E. Seim, and D. B. Percival (1993). Statistics of shear and turbulent dissipation profiles in random internal wave field. J. Phys. Oceanogr., 23, 1777-1799.

Kraichnan, R. (1968). Small-scale structure of a scalar field convected by turbulence. Phys. Fluids, 11, 945-953.

Lien, R. C., D. R. Caldwell, M. C. Gregg, and J. N. Moum (1995). Turbulence variability at the equator in the central Pacific at the beginning of the 1991-1993 El Nino. J. Geophys. Res., 100, 6881-6898.

Moum, J. N., and J. D. Nash (2009). Mixing measurements on an Equatorial ocean mooring. $J$. Atmos. Oceanic Technol., 26, 317-335.

Nash, J. D., D. R. Caldwell, M. J. Zelman, and J. N. Moum (1999). A thermocouple probe for high-speed temperature measurement in the ocean. J. Atmos. Oceanic Technol., 16, 14741482.

Osborn, T. R., and C. S. Cox (1972). Oceanic fine structure. Geophys. Fluid Dyn., 3, 321-345.

Sanford, T.B., R.G. Drever, J.H. Dunlap and E.A. D'Asaro (1982), Design, operation and performance of an Expendable Temperature and Velocity Profiler (XTVP). University of Washington, Applied Physics Laboratory Technical Report APL-UW 8110: 164 p.

Sanford, T. B., R. G. Drever and J. H. Dunlap (1985). An acoustic Doppler and electromagnetic velocity profiler. J. Atmos. Oceanic Technol., 2, 110-124.

Sanford, T.B., J.H. Dunlap, J.A. Carlson, D.C. Webb, and J.B. Girton (2005). Autonomous velocity and density profiler: EM-APEX. Proceedings of the IEEE/OES Eighth Working 
Autonomous Microstructure_EM-APEX_Float 10/17/17 at 10:31 PM

Conference on Current Measurement Technology, IEEE Cat No. 05CH37650, ISBN: 0-78038989-1, 152-156.

Shcherbina, A. Y., M. A. Sundermeyer, E. Kunze, E. D’Asaro, G. Badin, D. Birch, A.-M. E. G. Brunner-Suzuki, J. Callies, B. T. Kuebel Cervantes, M. Claret, B. Concannon, J. Early, R. Ferrari, L. Goodman, R. R. Harcourt, J. M. Klymak, C. M. Lee, M.-P. Lelong, M. D. Levine, R.-C. Lien, A. Mahadevan, J. C. McWilliams, M. J. Molemaker, S. Mukherjee, J. D. Nash, T. Özgökmen, S. D. Pierce, S. Ramachandran, R. M. Samelson, T. B. Sanford, R. K. Shearman, E. D. Skyllingstad, K. S. Smith, A. Tandon, J. R. Taylor, E. A. Terray, L. N. Thomas, and J. R. Ledwell (2015). The LatMix summer campaign: Submesoscale stirring in the upper ocean. Bull. Amer. Meteor. Soc., 96, 1257-1279. doi: http://dx.doi.org/10.1175/BAMS-D-14-00015.1

Sommer, T., J. R. Carpenter, M. Schmid, R. G. Lueck, and F. Wuest (2013). Revisiting microstructure sensor responses with implications for double-diffusive fluxes. J. Atmos. Ocean. Technol., 30, 1907-1923.

Sundermeyer, M. A., D. A. Birch, J. R. Ledwell, M. D. Levine, S. D. Pierce, B. T. Kuebel Cervantes, B. Conannon, (2016). Dye Dispersion in the open ocean seasonal pycnocline at scales of 1-10 km and 1-6 days. In Preparation for submission to J. Phys. Oceanography. Vachon, P., and R. Lueck (1984). A small combined temperature-conductivity probe. Proc. 1984 STD Conf. and Workshop, San Diego, California, Marine Technology Society San Diego Section and MTS Oceanic Instrumentation Committee, 126-131.

Yamazaki, H. A., and R. Lueck (1990). Why oceanic dissipation rates are not lognormal. J. Phys. Oceanogr., 20, 1907-1918.

Yamazaki, H. (1990). Breakage models: lognormality and intermittency. J. Fluid Mech., 219, 181-193.

Yaglom, A. M. (1966). On the influence of fluctuations in energy dissipation on the turbulence characteristics in the inertial internal. Dokl. Akad. Nauk. SSSR, 166, 49-52. 

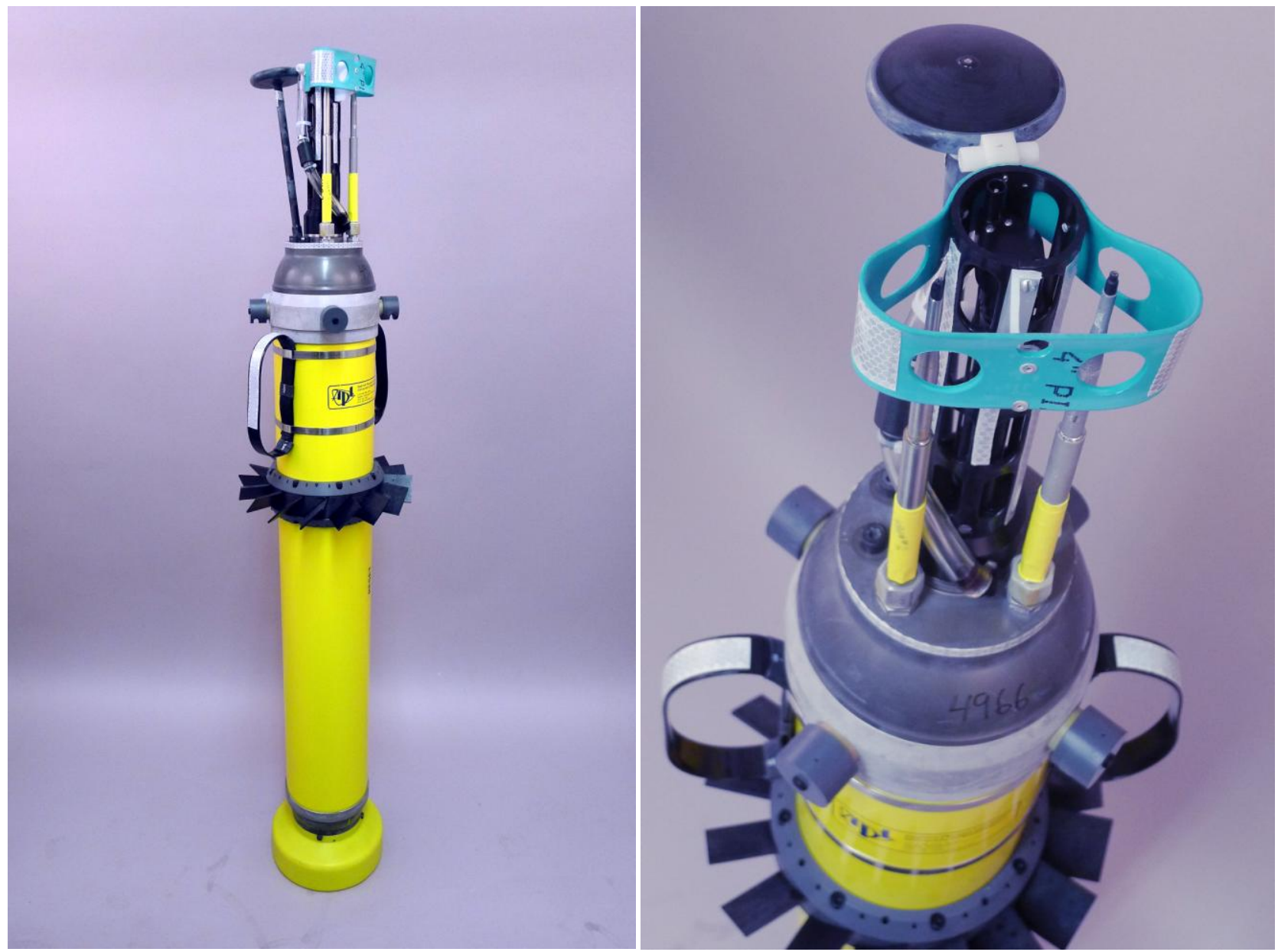

Figure 1: Photos of EM-APEX float with electrode collar and rotation vanes (left) with dual fastresponse temperature sensors FP-07 inside a guard (light green) and (right) a closer view of the FP-07 sensors, visible on black tips of metal shafts. Other components on the top of the float are the SBE41CP CTD in the center and the GPS/Iridium antenna. 


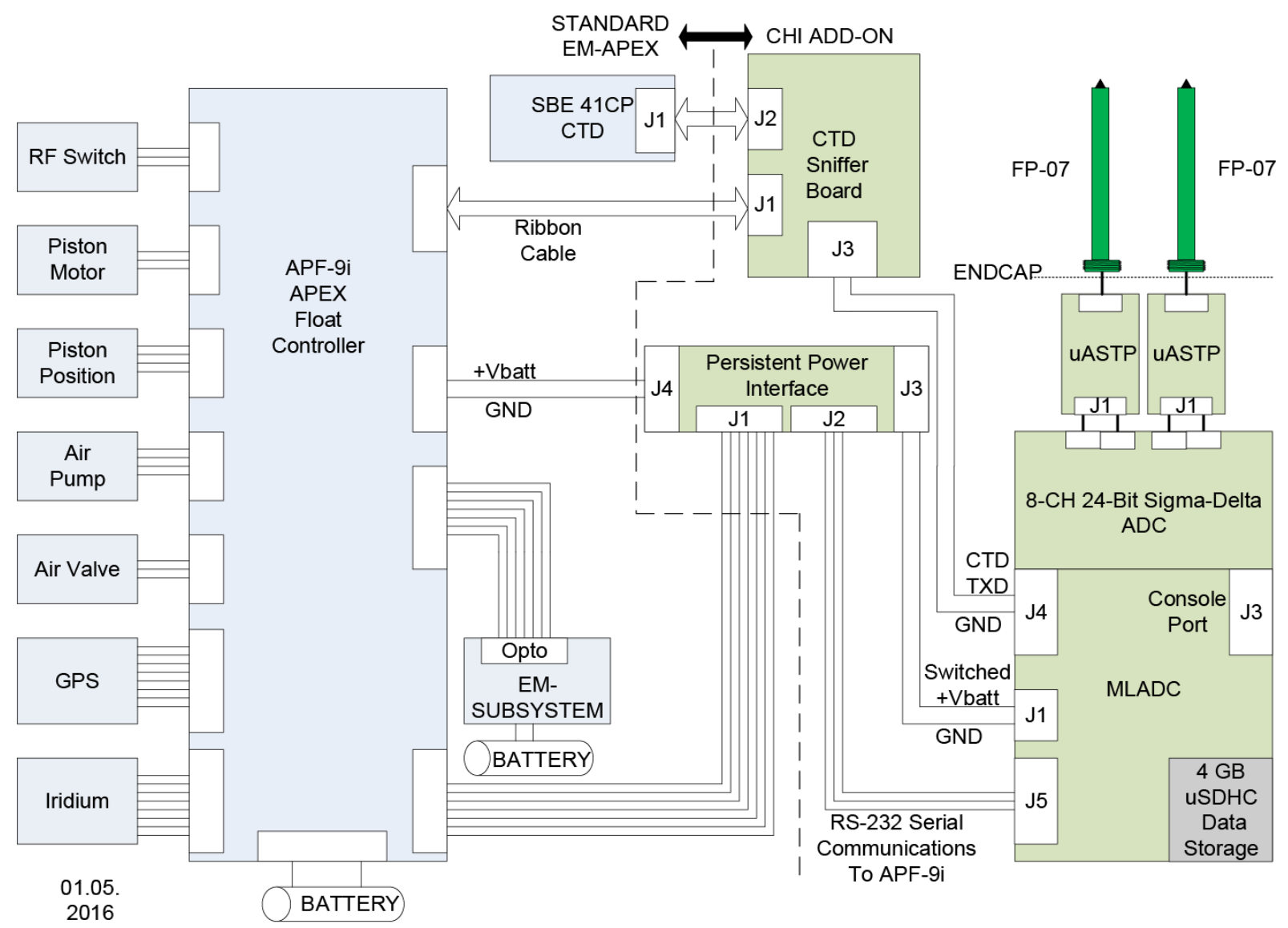

Figure 2: Block diagram of the microstructure EM-APEX float. The left side of the dashed line shows the standard configuration for an EM-APEX float (grey). The APF-9i APEX float controller runs the Sea-Bird CTD while using the air pump, air valve, and piston motor to control buoyancy. At the surface the APF-9i uses a single antenna with an RF switch for GPS position information and Iridium communications. The right side of the dashed line shows the components added to make an microstructure EM-APEX float (light green), including dual RSI FP-07 fast temperature probes (dark green), differential RSI pre-amps with a DC gain=x6 feeding into a 24-bit sigma-delta analog-to-digital converter and a dedicated microstructure EMAPEX controller. The APF-9i controls power to the $\chi$ system using the persistent power interface board. The $\chi$ controller on the APL-UW (MLADC) board uses the CTD sniffer circuit board to intercept the data stream from the CTD to the APF-9i to gather rise and fall rate data. The resulting microstructure EM-APEX data are stored on a MicroSD card. Reduced data are transmitted over the satellite link when on the surface, but the raw data are available only by removal of the storage card. 

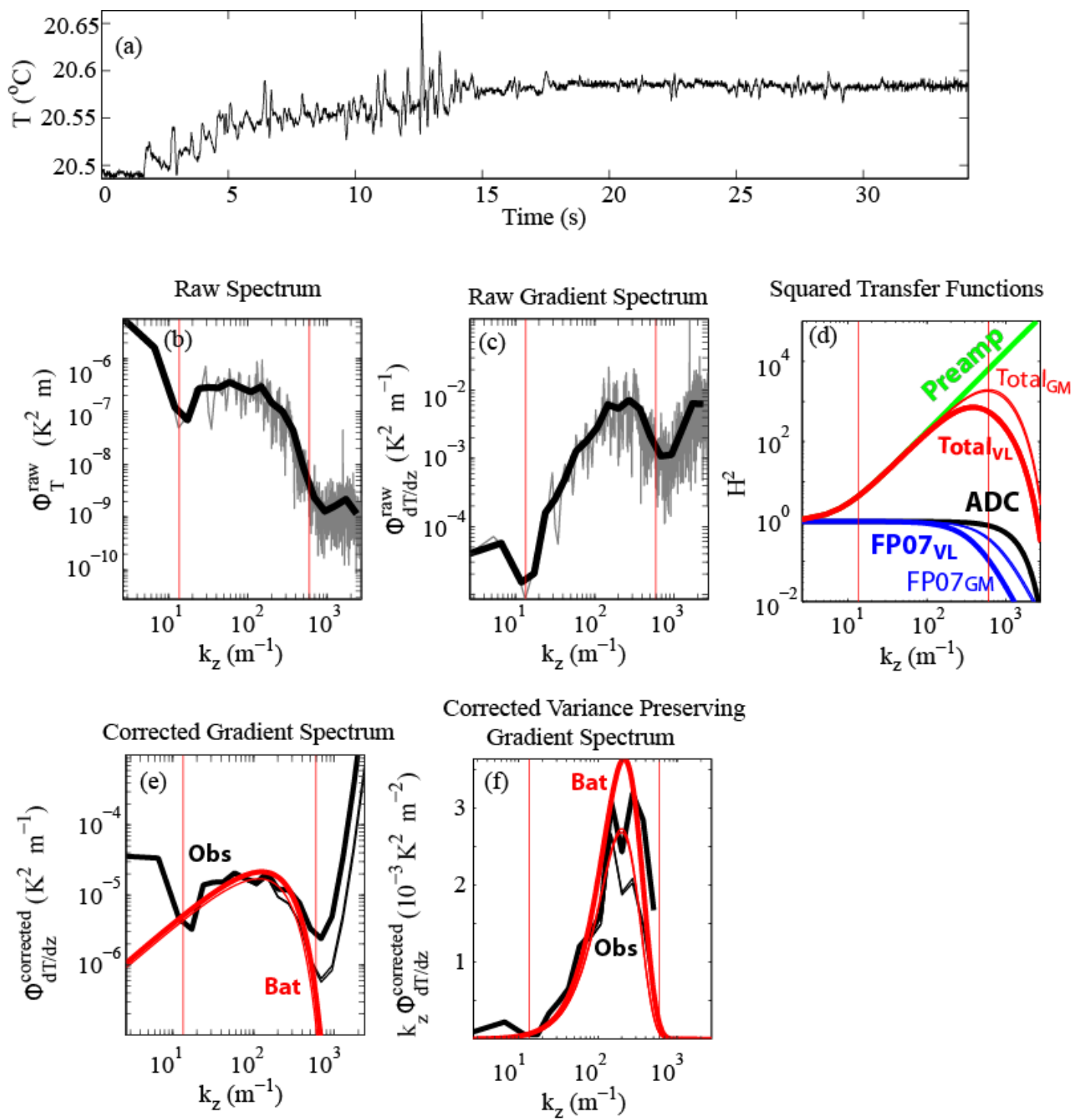

Corrected Variance Preserving

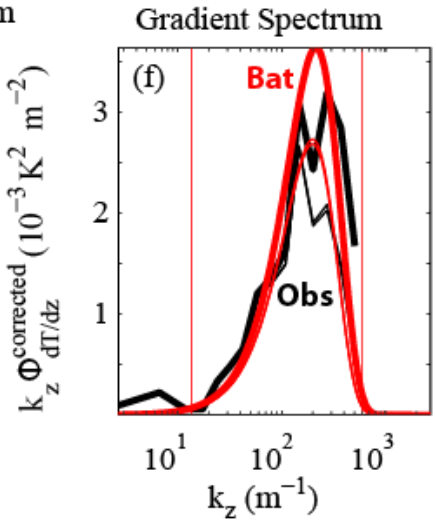

Figure 3: Examples of computed temperature variance dissipation rate $\chi$ from FP-07

measurements: (a) the time series of $35 \mathrm{~s}$ of temperature from one FP-07 sensor, (b) the observed raw temperature spectrum, (c) the observed raw spectrum of vertical gradient of temperature, (d) squared transfer functions of pre-amplifier ( $H_{\text {Preamp }}$, green curve labeled as Preamp), analog-todigital converter $\left(H_{\mathrm{ADC}}, \mathrm{ADC}\right)$ (black curve labeled as ADC), FP-07 sensor $\left(H_{\mathrm{FP} 07}\right.$, thick blue curve labeled as $\mathrm{FP} 07_{\mathrm{VL}}$ and thin blue curve labeled as $\mathrm{FP} 07_{\mathrm{GM}}$ ), and the product of the above three transfer functions (thick red curve labeled as Total ${ }_{\mathrm{VL}}$ and thin red curve labeled as Total $_{\mathrm{GM}}$ ), (e) observed temperature gradient spectrum corrected for transfer functions (thick and thin black 
curves labeled as Obs) and Batchelor temperature gradient spectrum (thick and thin red curve labeled as Bat), and (f) the variance preserving form of spectra shown in panel (e). The subscript 'VL' represents the use of FP-07 transfer functions proposed by Vachon and Lueck (1984). The subscript 'GM' represents the use of the transfer function suggested by Gregg and Meagher (1980), which is nearly identical to that of Sommer et al. (2013). Note that Batchelor spectra shown in panels (e) and (f) are computed using estimates of $\chi$ and $\varepsilon$ obtained from observed spectra. Thick and thin curves in panels (e) and (f) are computed using FP-07 transfer functions of Vachon and Lueck (1984) and Gregg and Meagher (1980), respectively. 


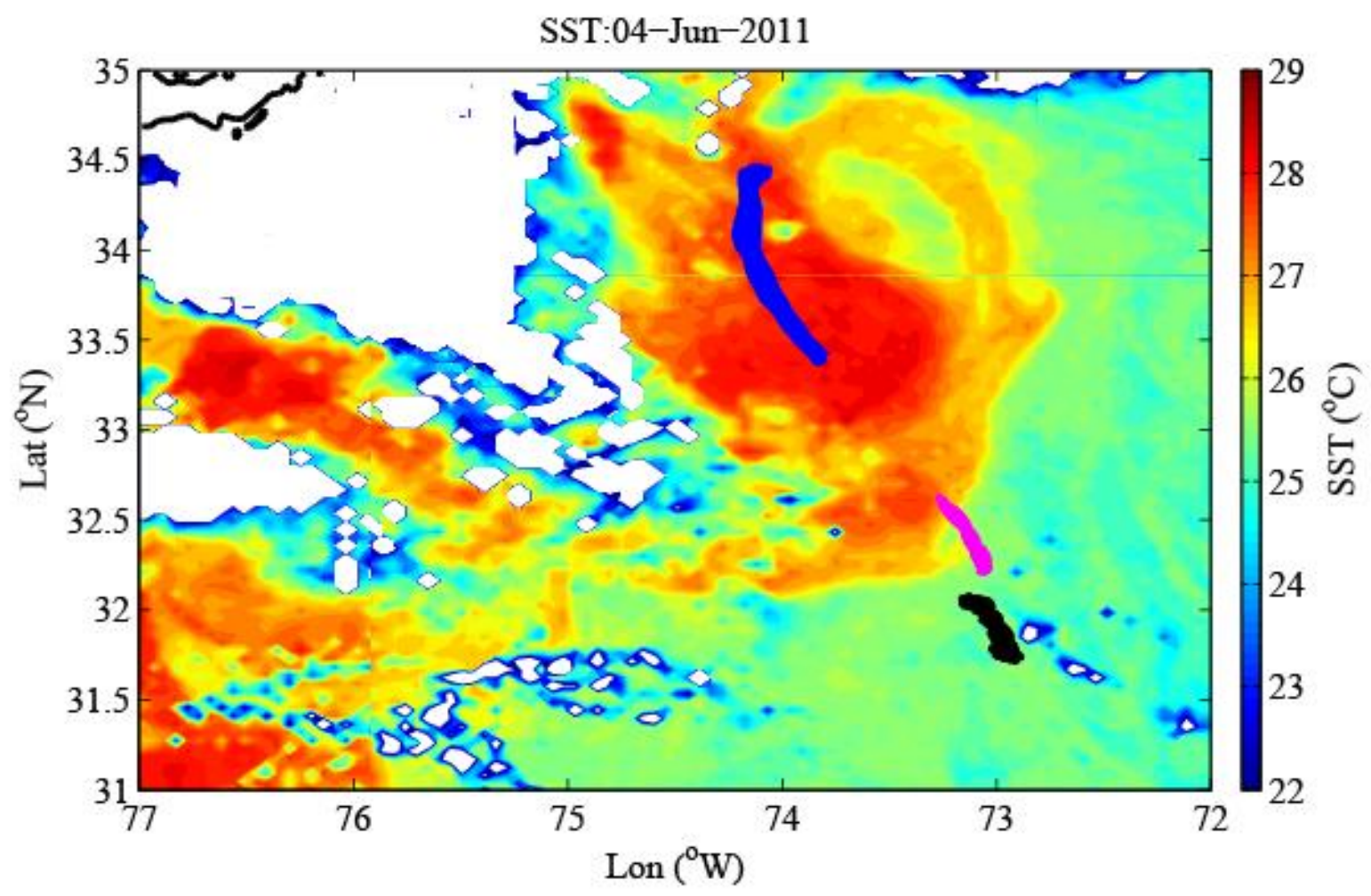

Figure 4: Float trajectories of three deployments during the LatMix experiment. Blue curves represent float trajectories on 6-10 June (D1), black curves on 13-16 June (D2), and pink curves on 17-20 June (D3). Background color shows the AVHRR image of SST on 4 June. 

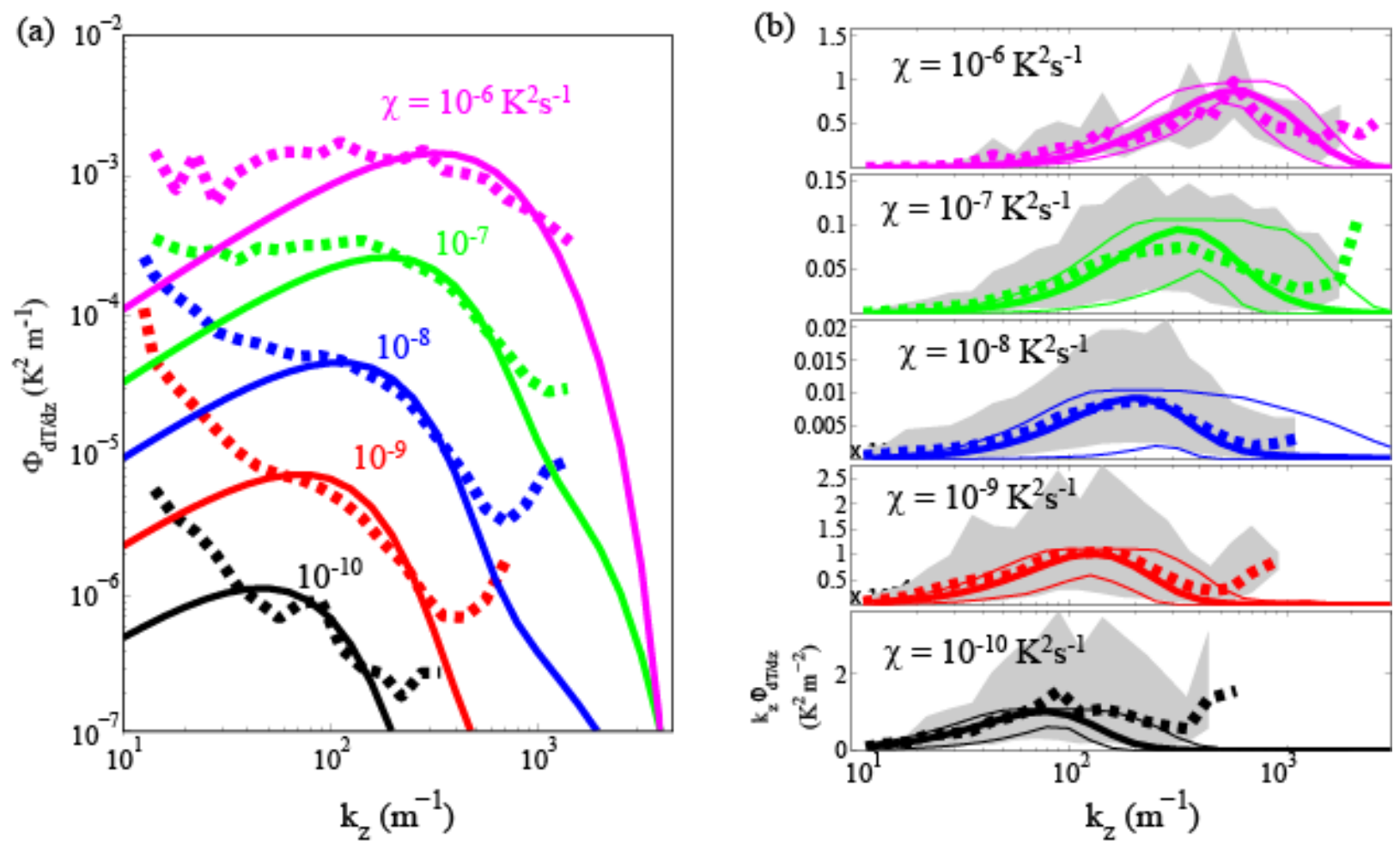

Fig. 5: (a) Comparison among observed temperature gradient spectra (dashed) with Batchelor spectra (solid) for $\chi$ of $10^{-10}-10^{-6} \mathrm{~K}^{2} \mathrm{~s}^{-1}$. (b) Similar to (a) but in variance preserving format. (b) Gray shadings represent $95 \%$ confidence interval of observed spectra, and thin curves represent 95\% confidence interval of Batchelor spectra. 
Vertical Profiles of Temporally Averaged $\chi$
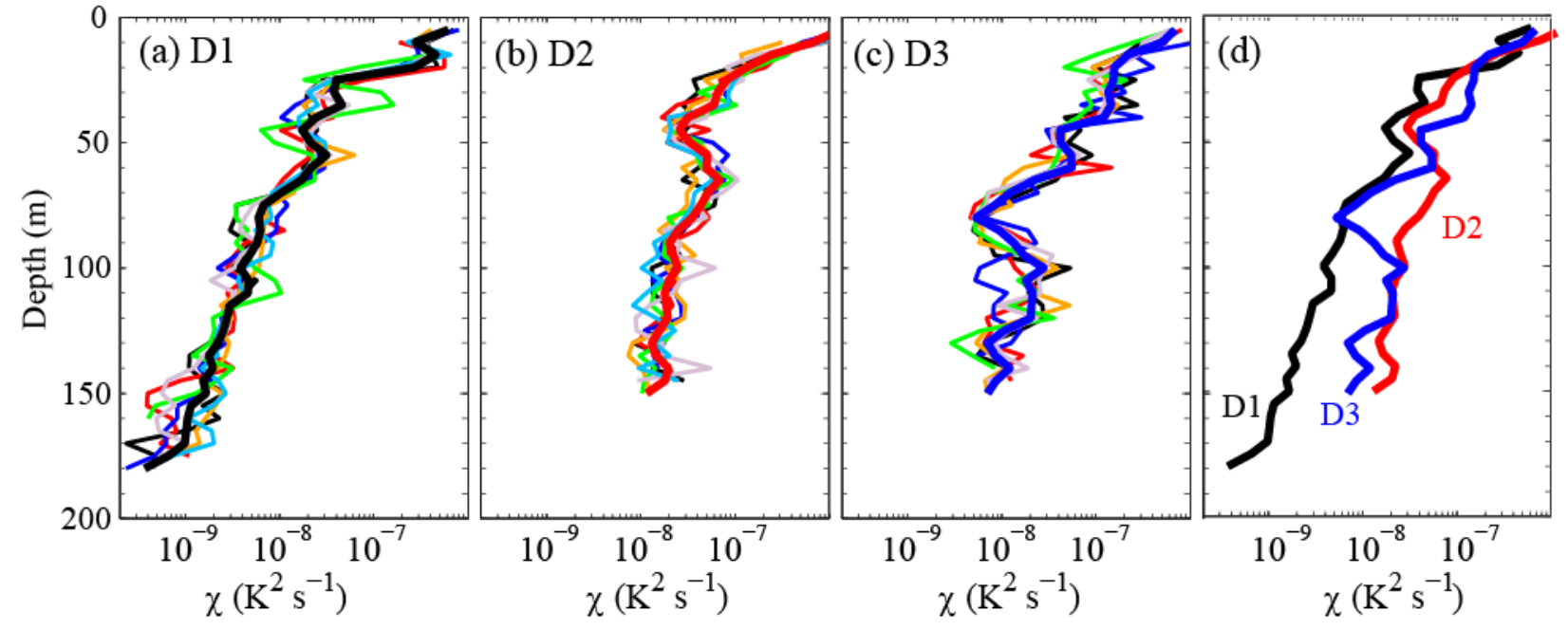

Figure 6. Vertical profiles of $\chi$ temporally averaged over three deployments of different floats $(a-c)$ and averaged over all floats (d). In panels (a)-(c) different thin colored curves represent results from different floats, and the thick curves represent averages over all floats. 


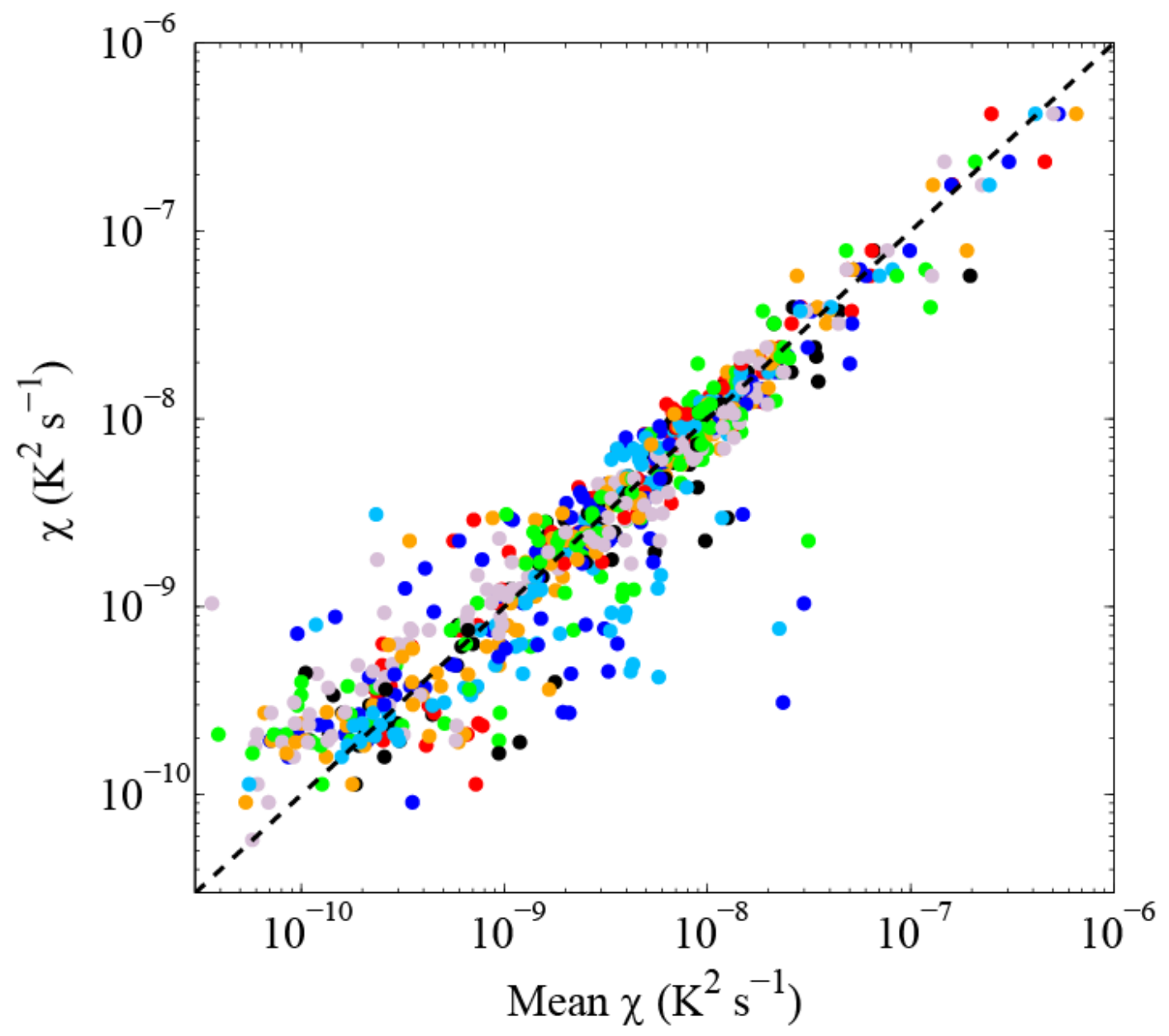

Fig. 7: Scatter plot of temporally averaged $\chi$ over each deployment period for different floats vs. temporally averaged $\chi$ over each deployment period and over all floats. Colors represent different floats. 


\section{Time Series of Depth Averaged $\chi$}
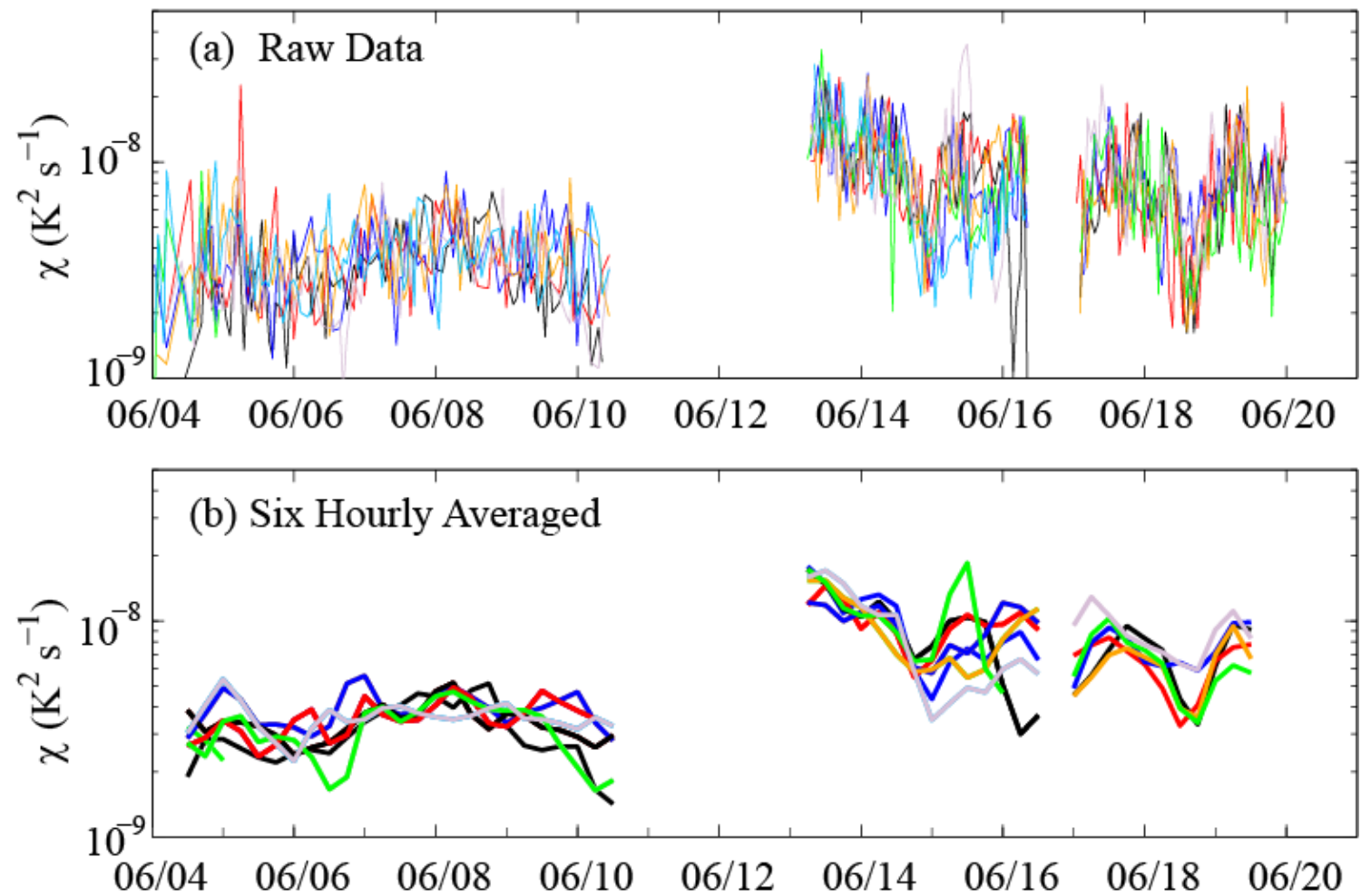

Fig. 8: (a) Time series of depth averaged $\chi$ from all floats, (b) six-hourly averaged values. 

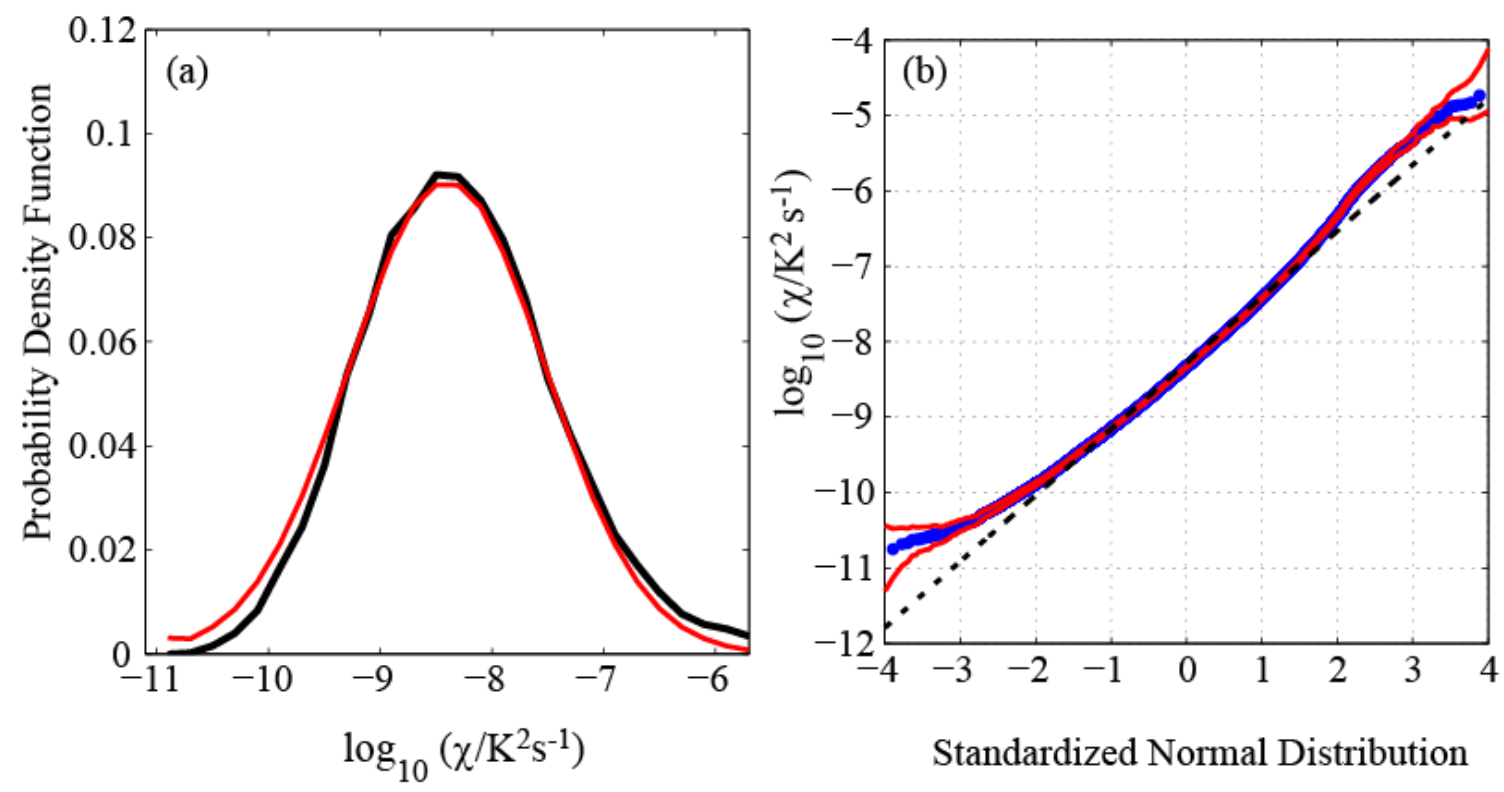

Fig. 9: (a) Probability density function of the ensemble of $\log _{10}(\chi)$ observations from all floats (black curve) compared with that expected from a theoretical normal distribution of the observed mean (-8.3) and standard deviation (0.9) (red curve). (b) Q-Q plot of $\log _{10}(\chi)$ vs. that of standardized normal distribution. The blue curve shows observed Q-Q structure. Red curves represent the $95 \%$ confidence interval assuming a normal distribution. The black dashed curve represents the theoretical normal distribution. 

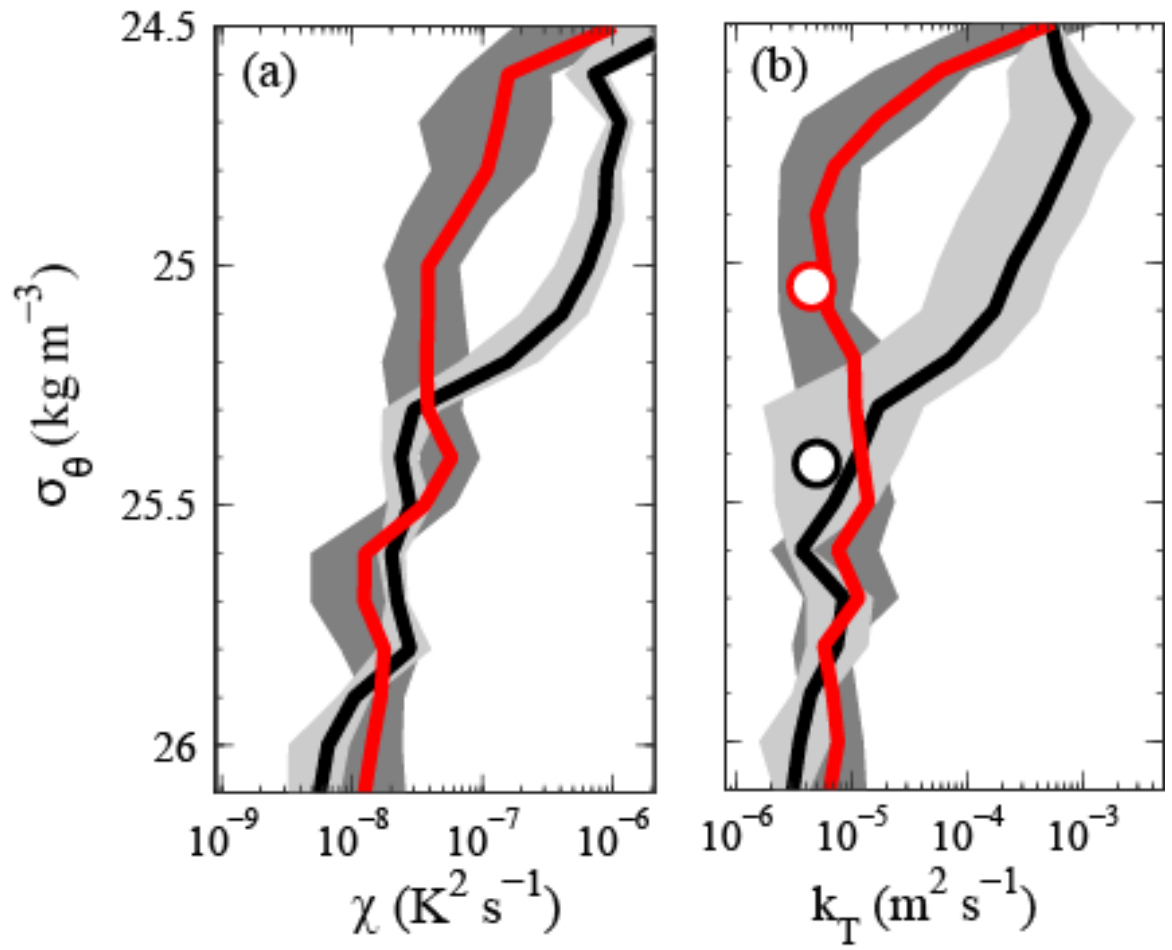

Figure 10. Profiles of (a) temperature variance diffusion rate $\chi$ and (b) turbulent temperature diffusivity $K_{T}$ temporally averaged over the weak straining period (black curve) and the moderate straining period (red curve), and averaged over all floats on isopycnal surfaces. Grey shadings represent $95 \%$ confidence intervals. Note that the weak straining period is between 4 June and 10 June during D1 (Fig. 4), and the moderate straining period is between 13 June and 20 June during D2 and D3. The black and red circles in panel (b) represent the diapcynal diffusivity averaged over the weak and moderate straining periods, respectively, estimated using tracer measurements at the respective potential densities of $25.42 \mathrm{~kg} \mathrm{~m}^{-3}$ and $25.04 \mathrm{~kg} \mathrm{~m}^{-3}$ (Shcherbina et al., 2015; Sundermeyer et al., 2016). 